PHYSICAL REVIEW A 93, 039901(E) (2016)

\title{
Erratum: Bogoliubov-wave turbulence in Bose-Einstein condensates [Phys. Rev. A 91, 053620 (2015)]
}

Kazuya Fujimoto and Makoto Tsubota

(Received 18 February 2016; published 2 March 2016)

DOI: 10.1103/PhysRevA.93.039901

Our paper contains three trivial mistakes that do not change the conclusion of this work.

The first one is the mathematical expression of Eq. (11). The correct expression is given by

$$
i \hbar \frac{\partial}{\partial t} \bar{\phi}(\boldsymbol{k})=\frac{\partial H}{\partial \bar{\phi}^{*}(\boldsymbol{k})} .
$$

The second one is the mathematical expression of Eq. (26), whose correct expression is as follows:

$$
i \hbar \frac{\partial}{\partial t} b(\boldsymbol{k})=\frac{\partial H}{\partial b^{*}(\boldsymbol{k})} .
$$

The third one is the value on the vertical axis for $C_{\mathrm{b}}(k)$ and $C_{\mathrm{d}}(k)$ in Fig. 4. The modified figure is Fig. 1 in this paper.
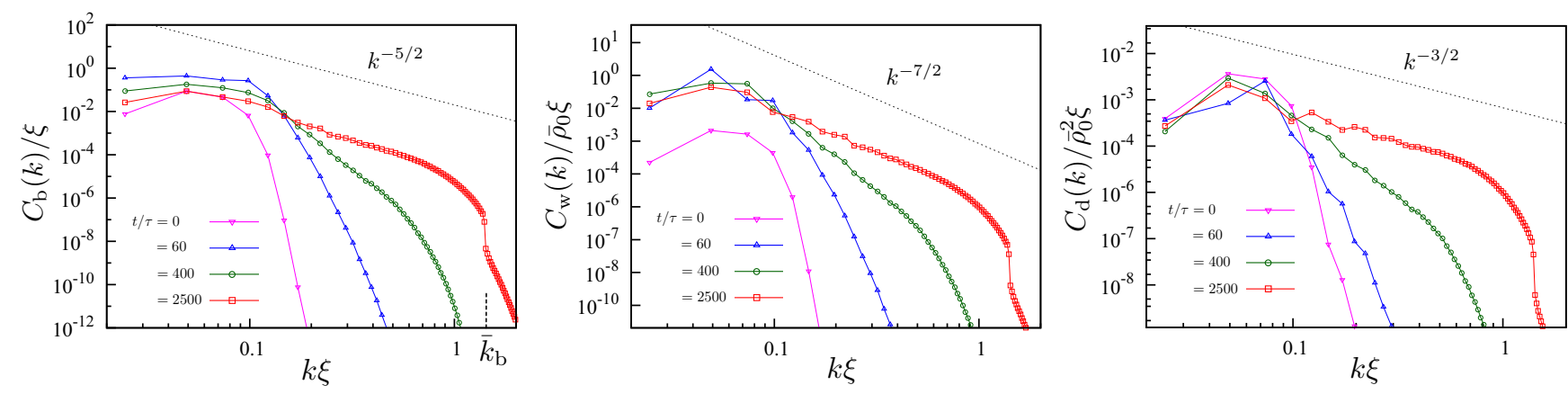

FIG. 1. Time development of the spectra for the Bogoliubov-wave distribution $C_{\mathrm{b}}$, the macroscopic wave function $C_{\mathrm{w}}$, and the density distribution $C_{\mathrm{d}}$. The value on the vertical axis for $C_{\mathrm{b}}(k)$ and $C_{\mathrm{d}}(k)$ is modified. 